\title{
Reintervención Quirúrgica No Programada: un análisis de calidad de la atención
}

\author{
Unplanned Return to the Operating Room: an analysis of the quality of the health care
}

\author{
Germán Muranda $^{a}$, Eduardo Focacci ${ }^{\mathrm{b}}$, José Mena ${ }^{\mathrm{c}}$ Sandra Montedonico ${ }^{\mathrm{b}, \mathrm{d}}$
}

\author{
aBecado, Programa de Formación de Cirugía Pediátrica de la Universidad de Valparaíso. Valparaíso, Chile \\ bServicio de Cirugía Pediátrica, Hospital Carlos Van Buren. Valparaíso, Chile \\ 'Servicio de Cirugía Pediátrica, Hospital Gustavo Fricke, Viña del Mar, Chile \\ dPrograma de Formación de Cirugía Pediátrica de la Universidad de Valparaíso. Valparaíso, Chile
}

Recibido: 30 de diciembre de 2019; Aceptado: 3 de agosto de 2020

¿Qué se sabe del tema que trata este estudio?

Las reintervenciones quirúrgicas no programadas son un indicador de calidad de los servicios quirúrgicos en Chile desde el año 2012. El análisis de las mismas en el seno del equipo tratante puede ayudar a mejorar la calidad de atención.

\section{¿Qué aporta este estudio a lo ya conocido?}

Este estudio describe las reintervenciones quirúrgicas no programadas en un servicio de cirugía pediátrica en un período de 5 años tanto en patología electiva como de urgencia; analiza las causas de las mismas y propone alternativas de optimización de su manejo.

\section{Resumen}

Una Reintervención Quirúrgica No Programada (RQNP) es aquella cirugía no planificada que se realiza durante los primeros 30 días como consecuencia de una cirugía primaria. En Chile, el análisis y la tasa de RQNP son un indicador de calidad. Objetivo: describir y analizar las RQNP en pediatría. Pacientes y Método: Estudio observacional de corte transversal. Se revisaron los registros clínicos de los pacientes pediátricos sometidos a RQNP en el Hospital Carlos Van Buren en un período de 5 años. Se analizó su incidencia, indicaciones y causas que se clasificaron en 1) causas atribuibles a la técnica quirúrgica; 2) causas relacionadas al tratamiento; 3) patología propia del paciente y 4) otras causas. Se analizó además el cumplimiento de reuniones de análisis de RQNP. Resultados: Se efectuaron 23 RQNP de un total de 5.503 cirugías en 5 años $(0,42 \%)$. Hubo 11 RQNP de 3.434 cirugías electivas realizadas y 12 RQNP de 2069 cirugías de urgencia realizadas $(0,32 \% v / s, 0,58 \%$ respectivamente, $\mathrm{p}=\mathrm{NS})$. Hubo $2 \mathrm{RQNP}$ en los 82 recién nacidos operados en el período $(2,43 \%, \mathrm{p}<0,01)$. En todos los casos se realizaron reuniones de análisis de RQNP. En 18 de los 23 pacientes sometidos a RQNP se encontró una causa atribuible a la técnica o planificación quirúrgica. Conclusiones: Las RQNP son poco frecuentes en pediatría excepto en el período neonatal. Se da total cumplimiento a la normativa nacional de reunión de análisis luego de una RQNP que indican que las causas son mayoritariamente atribuibles a la técnica o planificación quirúrgica.
Palabras clave:

Reintervención

Quirúrgica No

Programada;

Indicador de Calidad;

Cirugía Segura;

Cirugía Pediátrica

Correspondencia:

Germán Muranda

german.muranda@gmail.com 


\section{Abstract}

An Unplanned Return to the Operating Room (UROR) is an unplanned surgery performed during the first 30 days as a result of primary surgery. In Chile, the analysis and the UROR rate are quality indicators. Objective: to describe and analyze UROR in a pediatrics. Patients and Method: Observational cross-sectional study. The clinical records of pediatric patients undergoing UROR at the Hospital Carlos Van Buren over 5 years were reviewed. The incidence, indications, and causes of UROR were analyzed. The causes of UROR were classified as 1) causes attributable to surgical technique, 2) treatment-related causes, 3 ) the patient pathology, and 4) other causes. In addition, the observance of the case review meetings after an UROR was analyzed. Results: 23 UROR out of 5,503 surgeries were performed in 5 years, $(0.42 \%)$. There were 11 UROR out of 3,434 elective surgeries and 12 UROR out of 2,069 emergency ones $(0.32 \% v / s \quad 0.58 \%$ respectively, $p=$ NS $)$. There were 2 UROR out of 82 surgeries in newborns, $(2.43 \%, p<0.01)$. After every UROR, a case review meeting was held. In 18 out of the 23 patients who underwent UROR (78\%), the cause was attributable to the surgical technique or planning. Conclusions: UROR is rare in pediatric surgery, except for the newborn period. Case review meetings are held after every UROR case, according to the national guidelines. The causes of UROR are mostly attributable to the surgical technique or planning.

\section{Keywords:}

Unplanned Return to the Operating Room; Quality Indicator; Safe Surgery; Pediatric Surgery

\section{Introducción}

En los últimos años, la calidad en la atención de salud ha cobrado gran relevancia, exigiéndose los mejores resultados en las prestaciones de salud, que son periódicamente evaluados. Algunos indicadores de calidad son: el tiempo de hospitalización, la tasa de rehospitalización luego del alta, las encuestas de satisfacción del paciente y la morbilidad y mortalidad durante los primeros 30 días postoperatorios. ${ }^{1}$ En Chile, la calidad de la atención y seguridad del paciente está regulada por una norma ministerial que entró en vigencia en Octubre del año 2012 y que comprende: el reporte de eventos adversos y eventos centinela, la aplicación de la lista de chequeo para la seguridad de una cirugía, el análisis de reoperaciones quirúrgicas no programadas, la prevención de enfermedad tromboembólica en pacientes quirúrgicos, la prevención de úlceras por presión en pacientes hospitalizados, el reporte de caídas de pacientes hospitalizados y el programa de prevención de infecciones asociadas a la atención en salud ${ }^{2}$. En cirugía, una de las herramientas propuestas como indicador de calidad es el análisis de las reintervenciones quirúrgicas no programadas (RQNP) $)^{3}$, que es aquella intervención quirúrgica no planificada que se le realiza a un paciente ya operado, como consecuencia de la cirugía primaria, dentro de los primeros 30 días de postoperado ${ }^{4}$. En Chile, el Ministerio de Salud exige el análisis del 100\% de las RQNP en el seno del equipo de salud que trató al paciente y que la tasa de RQNP sea menor a un $2 \%$ o que disminuya un $10 \%$ de la línea base (acumulada a diciembre del año anterior). ${ }^{4} \mathrm{El} \mathrm{ob}-$ jetivo del presente trabajo es realizar una descripción y análisis de las RQNP en un servicio de Cirugía Pediátrica durante un período de 5 años.

\section{Pacientes y Método}

\section{Diseño}

Estudio observacional de corte transversal que incluyó a todos los pacientes menores de 15 años sometidos a reintervenciones quirúrgicas no programadas en el Hospital Carlos Van Buren de Valparaíso, entre los años 2014 y 2018. El presente estudio contó con la autorización del Comité Etico Científico del Servicio de Salud Valparaíso - San Antonio (Ord.: 2690 del 28/12/17).

\section{Definiciones}

Los Grupos Relacionados por el Diagnóstico (GRD) son un sistema de clasificación de pacientes que egresan de un hospital a partir de la información contenida en la ficha clínica. El uso de GRD permite a los hospitales monitorear la utilización de los recursos y la calidad del servicio, al relacionar los datos demográficos, diagnósticos y procedimientos de los pacientes con los costos involucrados en su cuidado ${ }^{5,6}$. A modo de referencia el Hospital Clínico de la Universidad de Chile tiene un peso medio GRD de 0,9929 en un período de 10 años. ${ }^{5}$ El Hospital Carlos Van Buren es un hospital de alta complejidad con un peso medio GRD de 1.0207 al año 2018.

Se define una RQNP como la realización de una intervención quirúrgica no planificada a un paciente ya operado, como consecuencia de la cirugía primaria, dentro de los primeros 30 días de la intervención. ${ }^{4}$ Se incluyeron todos los pacientes pediátricos operados de una patología de cirugía general, cirugía digestiva, cirugía neonatal, urología y cirugía plástica, tanto electivas como urgencias. Se excluyeron todos los pacientes que habiendo sido intervenidos dentro de los primeros 
30 días de una cirugía, ésta no se realizó como consecuencia de la cirugía primaria. También se excluyeron aquellos pacientes operados de patología neuroquirúrgica, otorrinolaringológica, oftalmológica y traumatológica ya que no son realizadas por médicos especialistas en cirugía pediátrica.

\section{Procedimientos}

Se revisaron los registros clínicos de todos los pacientes que cumplían con los criterios de inclusión, aportados por la Unidad de Calidad de nuestro hospital, así como las actas de las reuniones de análisis de dichas reintervenciones realizadas en el Servicio de Cirugía Pediátrica, aportadas por la jefatura del Servicio. Con la información obtenida se calculó la tasa de RQNP, se identificó las RQNP analizadas en reunión clínica, se determinaron las cirugías primarias y si eran electivas o de urgencia, los tipos de reintervenciones realizadas y la indicación de reintervención. Las causas de las reintervenciones se clasificaron en 1) causas atribuibles a la técnica quirúrgica; 2) causas relacionadas al tratamiento; 3) patología propia del paciente, y 4) otras causas, según proponen Kroon y cols. ${ }^{7}$ Se realizaron comparaciones de proporciones con el método de chi-cuadrado y $t$ de student y se consideró una diferencia significativa cuando $p$ era menor de 0,05 .

\section{Resultados}

Entre los años 2014 y 2018 se efectuaron un total de 9.598 cirugías en menores de 15 años. De este total se excluyeron los pacientes sometidos a cirugías neuroquirúrgicas, 838 pacientes; otorrinolaringológicas, 1.822 pacientes; oftalmológicas, 351 pacientes y traumatológicas, 1.084 pacientes. El total de pacientes analizados fueron 5.503. De este grupo, 309 pacientes fueron sometidos a una o más de una reintervención quirúrgica dentro de los 30 días siguientes a una intervención en el período estudiado; 23 de estos 309 co- rrespondían a una RQNP (0,42\% del total de pacientes operados). En la totalidad de los casos sometidos a RQNP se realizó una reunión de análisis de los mismos entre los cirujanos miembros del Servicio de Cirugía Pediátrica del hospital. Del total de cirugías realizadas, 3434 fueron cirugías electivas, entre las cuales hubo 11 RQNP (0,32\%). Las cirugías de urgencias fueron 2069, entre las cuales hubo 12 casos de RQNP (0,58\%). A pesar de que las RQNP luego de una cirugía de urgencia casi duplican a las cirugías electivas, estas diferencias no fueron significativas $(\mathrm{p}=0,1475)$ (tabla 1$)$. De manera complementaria, se realizó un promedio de los porcentajes de RQNP con intervalo de confianza del 95\% para las desviaciones de los promedios y se comparó la proporción de RQNP de urgencia y electivas del total del período estudiado siendo el valor de $\mathrm{p}=0,1939$.

La tabla 1 muestra los casos de RQNP de cada año analizado, destacando el bajo número de RQNP en los dos primeros años analizados.

Entre los años 2014 y 2018 se operaron 82 recién nacidos. De ellos, hubo 2 RQNP (2,43\%), lo cual es una proporción significativamente mayor en comparación tanto al total de RQNP $(0,42 \%)$ como a las RQNP cuya cirugía primaria fue una urgencia $(0.58 \%)$ para el período estudiado $(\mathrm{p}=0,0069$ y $\mathrm{p}=0,0410$, respectivamente).

Del total de pacientes reoperados, en 4 casos los niños ya se habían dado de alta y hubo que rehospitalizarlos para su reintervención.

Las tablas 2 y 3 muestran las RQNP cuando la primera cirugía fue electiva y de urgencia, respectivamente. Se detallan la edad, el diagnóstico preoperatorio inicial, la cirugía primaria realizada, la indicación de reoperación, la reintervención realizada y sus causas.

Dentro de las indicaciones de RQNP, destacan peritonitis/absceso intraabdominal en cinco casos, síndrome compartimental abdominal en cuatro casos, obstrucción intestinal mecánica en tres pacientes y evisceración en dos.

Tabla 1. Número y porcentaje de Reintervenciones Quirúrgicas No Programadas en relación al tipo de cirugía

\begin{tabular}{lccc}
\hline & Cirugía de urgencia & Cirugía electiva & Total \\
\hline Año 2014 & $1 / 419$ & $2 / 827$ & $3 / 1.246$ \\
Año 2015 & $1 / 441$ & $1 / 752$ & $2 / 1.193$ \\
Año 2016 & $3 / 434$ & $4 / 561$ & $7 / 995$ \\
Año 2017 & $4 / 427$ & $1 / 622$ & $5 / 1.049$ \\
Año 2018 & $3 / 348$ & $3 / 672$ & $6 / 1.020$ \\
Total 5 años & $12 / 2069(0,58 \%)^{*}$ & $11 / 3434(0,32 \%)$ & $23 / 5.503(0,42 \%)$ \\
& (media anual: 2,$4 ; 95 \% \mid C: 1,23-3,57)$ & (media anual: 2,$2 ; 95 \% \mid C: 1,06-3,34)$ & (media anual: 4,$6 ; 95 \%$ IC: $2,78-6,41)$ \\
\hline$*$
\end{tabular}

${ }^{*} p=0,1475$ (NS) versus total de cirugías electivas. 
Tabla 2. Resumen del total de pacientes sometidos a Reintervención Quirúrgica No Programada (RQNP) cuando la cirugía primaria fue electiva

\begin{tabular}{|c|c|c|c|c|c|c|}
\hline Caso & Edad & $\begin{array}{l}\text { Diagnóstico } \\
\text { preoperatorio }\end{array}$ & Cirugía primaria & $\begin{array}{l}\text { Indicación de } \\
\text { reoperación }\end{array}$ & RQNP & Causas de RQNP§ \\
\hline 1 & $\begin{array}{l}1 \text { mes } \\
11 \text { días }\end{array}$ & $\begin{array}{l}\text { Trastorno de } \\
\text { deglución }\end{array}$ & $\begin{array}{l}\text { Gastrostomía de } \\
\text { Stamm }\end{array}$ & $\begin{array}{l}\text { Disfunción de } \\
\text { gastrostomía }\end{array}$ & $\begin{array}{c}\text { Recambio } \\
\text { de sonda de } \\
\text { gastrostomía }\end{array}$ & $\begin{array}{l}\text { Tto: rotura del balón de } \\
\text { sonda por mal manejo de } \\
\text { enfermería }\end{array}$ \\
\hline 2 & $\begin{array}{l}1 \text { mes } \\
14 \text { días }\end{array}$ & $\begin{array}{l}\text { Probable Enfermedad } \\
\text { de Hirschsprung }\end{array}$ & $\begin{array}{l}\text { Colostomía en asa, } \\
\text { biopsia rectal }\end{array}$ & $\begin{array}{l}\text { Evisceración } \\
\text { intestinal }\end{array}$ & $\begin{array}{l}\text { Laparotomía } \\
\text { Exploradora. }\end{array}$ & $\begin{array}{l}\text { Técnica: Colostomía en asa } \\
\text { sin puente aponeurótico } \\
\text { Tto: instalación precoz y } \\
\text { manipulación de bolsa de } \\
\text { colostomía }\end{array}$ \\
\hline 3 & 1 año & $\begin{array}{l}\text { Enfermedad de } \\
\text { Hirschsprung }\end{array}$ & $\begin{array}{l}\text { Descenso endorrectal } \\
\text { abdominoperineal }\end{array}$ & $\begin{array}{c}\text { Dehiscencia } \\
\text { enteroanastomosis }\end{array}$ & Aseo, colostomía & Técnica: Mesenterio tenso \\
\hline 4 & 1 año & Onfalocele gigante & $\begin{array}{l}\text { Plastía (rotación } \\
\text { colgajos) }\end{array}$ & Necrosis de piel & $\begin{array}{l}\text { Aseo, drenaje } \\
\text { hematoma }\end{array}$ & $\begin{array}{c}\text { Técnica: diseño de colgajo } \\
\text { con poca vascularización. No } \\
\text { se dejaron drenajes }\end{array}$ \\
\hline 5 & 1 año & $\begin{array}{l}\text { 1. Intestino corto } \\
\text { 2. Trombosis venosa }\end{array}$ & $\begin{array}{l}\text { Instalación de CVC } \\
\text { (punción en cava } \\
\text { superior bajo visión } \\
\text { toracoscópica) }\end{array}$ & $\begin{array}{l}\text { Hidrotórax } \\
\text { masivo (nutrición } \\
\text { parenteral) }\end{array}$ & $\begin{array}{l}\text { Drenaje pleural, } \\
\text { aseo }\end{array}$ & $\begin{array}{c}\text { Técnica: variación de técnica } \\
\text { descrita sin experiencia } \\
\text { previa }\end{array}$ \\
\hline 6 & 5 años & Criptorquidia izquierda & Descenso testicular & $\begin{array}{l}\text { Evisceración } \\
\text { testicular }\end{array}$ & Aseo, resutura & $\begin{array}{c}\text { Técnica: Sutura/curación } \\
\text { insuficientes }\end{array}$ \\
\hline 7 & 6 años & $\begin{array}{l}\text { Hernia inguinal } \\
\text { bilateral }\end{array}$ & $\begin{array}{l}\text { Hernioplastía bilateral } \\
\text { (técnica de Burnia) }\end{array}$ & $\begin{array}{c}\text { Peritonitis } \\
\text { (uroperitoneo) }\end{array}$ & $\begin{array}{l}\text { Laparotomía } \\
\text { Exploradora }\end{array}$ & $\begin{array}{c}\text { Técnica: técnica nueva } \\
\text { con cirujanos con poca } \\
\text { experiencia en dicha técnica }\end{array}$ \\
\hline 8 & 10 años & $\begin{array}{l}\text { Enfermedad de } \\
\text { Hirschsprung }\end{array}$ & $\begin{array}{l}\text { Descenso endorrectal } \\
\text { abdominoperineal }\end{array}$ & $\begin{array}{l}\text { Peritonitis por } \\
\text { dehiscencia }\end{array}$ & $\begin{array}{l}\text { Aseo, resutura, } \\
\text { ileostomía }\end{array}$ & Técnica: mesenterio tenso \\
\hline 9 & 10 años & $\begin{array}{c}\text { Adenopatía cervical } \\
\text { izquierda }\end{array}$ & Biopsia & $\begin{array}{l}\text { Infección herida } \\
\text { operatoria }\end{array}$ & Drenaje & $\begin{array}{l}\text { Otras: contaminación sin } \\
\text { etiología definida }{ }^{\dagger}\end{array}$ \\
\hline 10 & 11 años & Cáncer de tiroides & $\begin{array}{c}\text { Tiroidectomía más } \\
\text { vaciamiento ganglionar }\end{array}$ & $\begin{array}{l}\text { Hemorragia } \\
\text { postoperatoria }\end{array}$ & $\begin{array}{l}\text { Drenaje } \\
\text { hematoma } \\
\text { cervical }\end{array}$ & $\begin{array}{c}\text { Técnica: hemostasia } \\
\text { insuficiente } \\
\text { Patología: Tumor infiltrante }\end{array}$ \\
\hline 11 & 14 años & $\begin{array}{l}\text { 1. Cuerpo extraño } \\
\text { intestinal } \\
\text { 2. Cicatriz abdominal } \\
\text { antiestética } \\
\text { 3. Enfermedad de } \\
\text { Hirschsprung operada }\end{array}$ & $\begin{array}{c}\text { Laparotomía } \\
\text { Exploradora } \\
\text { (Hallazgo: Síndrome } \\
\text { multiadherencial } \\
\text { severo, Cuerpo extraño } \\
\text { a nivel de válvula } \\
\text { ileocecal) }\end{array}$ & $\begin{array}{c}\text { Dehiscencia } \\
\text { herida operatoria }\end{array}$ & $\begin{array}{c}\text { Resutura }+ \\
\text { drenaje seroma }\end{array}$ & $\begin{array}{c}\text { Técnica: abordaje } \\
\text { inadecuado (perforación } \\
\text { intestinal iatrogénica } \\
\text { al ingresar a la cavidad } \\
\text { peritoneal) }\end{array}$ \\
\hline
\end{tabular}

${ }^{\S}$ Cuando hay más de una causa asociada a la RQNP, éstas se ordenaron según relevancia para los autores. Tto: Tratamiento. ${ }^{\dagger}$ En el caso 9 , después de analizar los protocolos operatorios, indicaciones, tratamientos y evolución de los pacientes, no se encontró una causa clara de la contaminación.

En relación a las causas de RQNP en algunos casos se encontró más de una causa: de las 23 reintervenciones analizadas, en 18 casos la principal causa de RQNP fue atribuible a la técnica quirúrgica o a la planificación de la cirugía, seguida por una causa asociada al tratamiento (5 casos), la patología propia del paciente ( 5 casos) y otras causas ( 2 casos). En 6 casos, hubo más de una causa atribuible a la RNQP (tablas 2 у 3$)$.

\section{Discusión}

Un buen indicador de calidad en salud debe poseer varias cualidades: 1) Importancia: la información obtenida debe ser relevante; 2) Confiabilidad: sus resultados deben ser repetibles; 3) Factibilidad: la información que entrega el indicador debe ser factible de obtener y 4) Claridad: los resultados se deben entender con facilidad ${ }^{8}$. En concordancia con estas cualidades, el 
Tabla 3. Resumen del total de pacientes sometidos a Reintervención Quirúrgica No Programada (RQNP) cuando la cirugía primaria fue una urgencia

\begin{tabular}{|c|c|c|c|c|c|c|}
\hline Caso & Edad & $\begin{array}{l}\text { Diagnóstico } \\
\text { preoperatorio }\end{array}$ & Cirugía primaria & $\begin{array}{l}\text { Indicación de } \\
\text { reoperación }\end{array}$ & RQNP & Causas de RQNP§ \\
\hline 1 & 0 días & Gastrosquisis & Cierre primario & $\begin{array}{l}\text { Sd. } \\
\text { Compartimental }\end{array}$ & $\begin{array}{l}\text { Laparostomía } \\
\text { contenida }\end{array}$ & $\begin{array}{l}\text { Técnica: No se midió PIA } \\
\text { intraoperatoria }\end{array}$ \\
\hline 2 & 17 días & ECN & $\begin{array}{l}\text { Laparotomía } \\
\text { exploradora }\end{array}$ & $\begin{array}{l}\text { Sd. } \\
\text { Compartimental }\end{array}$ & $\begin{array}{l}\text { Laparostomía } \\
\text { contenida }\end{array}$ & $\begin{array}{l}\text { Técnica: No se laparostomizó } \\
\text { Patología: progresión de ECN }\end{array}$ \\
\hline 3 & 2 meses & $\begin{array}{l}\text { Invaginación } \\
\text { intestinal }\end{array}$ & $\begin{array}{l}\text { Laparotomía } \\
\text { exploradora }\end{array}$ & $\begin{array}{l}\text { Sd. } \\
\text { Compartimental }\end{array}$ & $\begin{array}{l}\text { Laparotomía } \\
\text { exploradora, } \\
\text { laparostomía } \\
\text { contenida }\end{array}$ & $\begin{array}{c}\text { Técnica: Tiempo operatorio } \\
\text { prolongado } \\
\text { Patología: shock séptico } \\
\text { asociado } \\
\text { Tto: sobrecarga de volumen } \\
\text { en reanimación }\end{array}$ \\
\hline 4 & 2 meses & $\begin{array}{l}\text { 1. Gastrosquisis } \\
\text { operada } \\
\text { 2. Obstrucción } \\
\text { intestinal }\end{array}$ & $\begin{array}{l}\text { Laparotomía } \\
\text { exploradora, } \\
\text { Gastrostomía de } \\
\text { Stamm }\end{array}$ & Peritonitis & $\begin{array}{l}\text { Laparotomía } \\
\text { exploradora }\end{array}$ & $\begin{array}{c}\text { Técnica: Pexia inadecuada } \\
\text { de gareta de gastrostomía a } \\
\text { pared abdominal }\end{array}$ \\
\hline 5 & 6 meses & $\begin{array}{l}\text { Peritonitis } \\
\text { apendicular }\end{array}$ & $\begin{array}{l}\text { Laparotomía } \\
\text { exploradora, aseo }\end{array}$ & $\begin{array}{l}\text { Obstrucción } \\
\text { intestinal }\end{array}$ & $\begin{array}{l}\text { Laparotomía } \\
\text { exploradora }\end{array}$ & $\begin{array}{c}\text { Técnica: abordaje insuficiente } \\
\text { Patología: plastrón } \\
\text { apendicular abscedado }\end{array}$ \\
\hline 6 & 1 año & ECN complicada & $\begin{array}{l}\text { Colostomía } \\
\text { Hartmann }\end{array}$ & $\begin{array}{l}\text { Sospecha de } \\
\text { progresión de } \\
\text { ECN }\end{array}$ & $\begin{array}{c}\text { Laparotomía } \\
\text { exploradora, } \\
\text { movilización de ostomía }\end{array}$ & $\begin{array}{l}\text { Técnica: intestino ostomizado } \\
\text { con mesenterio tenso }\end{array}$ \\
\hline 7 & 5 años & Apendicitis aguda & $\begin{array}{c}\text { Apendicectomía } \\
\text { abierta } \\
\text { (Hallazgos: apéndice } \\
\text { sano, adenitis } \\
\text { mesentérica) }\end{array}$ & Peritonitis & $\begin{array}{c}\text { Laparotomía } \\
\text { exploradora (aseo } \\
\text { peritoneal) }\end{array}$ & $\begin{array}{l}\text { Otras: infección por } \\
\text { E. coli multisensible } \\
\text { y de S. pyogenes }\end{array}$ \\
\hline 8 & 6 años & $\begin{array}{l}\text { Pleuroneumonia } \\
\text { derecha }\end{array}$ & $\begin{array}{l}\text { Pleurotomía, tubo } \\
\text { pleural }\end{array}$ & $\begin{array}{l}\text { Tubo pleural } \\
\text { tapado }\end{array}$ & $\begin{array}{l}\text { Recambio de tubo } \\
\text { pleural }\end{array}$ & $\begin{array}{c}\text { Tto: vaciamiento de trampa } \\
\text { de agua } \\
\text { (¿por volcamiento?) }\end{array}$ \\
\hline 9 & 7 años & $\begin{array}{l}\text { 1. Peritonitis } \\
\text { apendicular } \\
\text { 2. Hernia } \\
\text { diafragmática } \\
\text { derecha operada }\end{array}$ & $\begin{array}{l}\text { Laparotomía } \\
\text { exploradora } \\
\text { (Incisión de } \\
\text { Rocky-Davis) }\end{array}$ & $\begin{array}{l}\text { Obstrucción } \\
\text { intestinal }\end{array}$ & $\begin{array}{c}\text { Laparotomía } \\
\text { exploradora, } \\
\text { laparostomía contenida } \\
\text { (Hallazgo: Obstrucción } \\
\text { intestinal por } \\
\text { adherencias) }\end{array}$ & $\begin{array}{l}\text { Técnica: diagnóstico } \\
\text { preoperatorio errado } \\
\text { determina un abordaje } \\
\text { inadecuado }\end{array}$ \\
\hline 10 & 9 años & $\begin{array}{l}\text { Peritonitis } \\
\text { apendicular }\end{array}$ & $\begin{array}{l}\text { Laparotomía } \\
\text { exploradora }\end{array}$ & $\begin{array}{l}\text { Sd. } \\
\text { Compartimental }\end{array}$ & $\begin{array}{l}\text { Laparotomía } \\
\text { exploradora, } \\
\text { laparostomía } \\
\text { contenida }\end{array}$ & $\begin{array}{c}\text { Técnica: abordaje } \\
\text { insuficiente, cirugía de } \\
\text { madrugada con ayudante no } \\
\text { cirujano }\end{array}$ \\
\hline 11 & 11 años & $\begin{array}{l}\text { Apendicitis } \\
\text { flegmonosa }\end{array}$ & $\begin{array}{l}\text { Apendicectomía } \\
\text { abierta }\end{array}$ & $\begin{array}{l}\text { Absceso } \\
\text { intraabdominal }\end{array}$ & $\begin{array}{l}\text { Laparotomía } \\
\text { exploradora, } \\
\text { laparostomía } \\
\text { contenida }\end{array}$ & $\begin{array}{c}\text { Técnica: aseo peritoneal } \\
\text { insuficiente (coprolito libre) } \\
\text { Tto: apéndice con perforación } \\
\text { traumática sin antibiótico } \\
\text { postoperatorio }\end{array}$ \\
\hline 12 & 13 años & Apendicitis catarral & $\begin{array}{l}\text { Apendicectomía } \\
\text { abierta }\end{array}$ & $\begin{array}{l}\text { Obstrucción } \\
\text { intestinal }\end{array}$ & $\begin{array}{c}\text { Laparotomía } \\
\text { exploradora } \\
\text { (Hallazgo: plastrón } \\
\text { apendicular abscedado) }\end{array}$ & $\begin{array}{l}\text { Patología: apendicitis más } \\
\text { pancolitis }\end{array}$ \\
\hline
\end{tabular}

${ }^{\S}$ Cuando hay más de una causa asociada a la RQNP, éstas se ordenaron según relevancia para los autores. PIA: Presión intraabdominal. ECN: Enterocolitis necrotizante. Tto: Tratamiento. 
seguimiento de las RQNP es un indicador valioso y útil por varias otras razones: es más frecuente que otros indicadores, como la mortalidad; puede ocurrir después de prácticamente cualquier procedimiento quirúrgico $y$, por lo tanto, es ampliamente aplicable; es un indicador no discrecional, es decir, el paciente sólo se reintervendrá cuando sea realmente necesario; y es de fácil seguimiento utilizando datos administrativos. Los resultados de nuestro estudio indican que la incidencia de RQNP en nuestro medio es baja, encontrándose por debajo de lo sugerido por el MINSAL que es de un 2\%. En cirugía de adultos, distintos autores reportan una incidencia que fluctúa entre un 0,6 y 9,4\% $\%^{3,9,10-12}$.

En cirugía pediátrica existen pocos estudios publicados. Ramírez y cols. ${ }^{13}$ reportan una incidencia de $1,8 \%$ de RQNP considerando solamente las reintervenciones después de una cirugía abdominal. Kulaylat y cols. ${ }^{14}$ analizaron los datos de las readmisiones en pacientes operados del Programa Nacional de Mejoramiento de la Calidad Quirúrgica Pediátrica (NSQIPP) del American College of Surgeons y encontraron una tasa de reoperación en cirugía general pediátrica del $0,88 \%$. Boo y cols ${ }^{15}$. encontraron una incidencia de RQNP de 3,5\%. Es esperable suponer que una cirugía de urgencia tenga una mayor probabilidad de complicarse y de requerir una reoperación que una cirugía electiva; esto fue lo que encontró Guevara y cols. ${ }^{12}$ en un estudio de cohorte en adultos. Nuestros resultados muestran que no existen diferencias en las tasas de RQNP luego de una cirugía de urgencia versus una cirugía electiva en la edad pediátrica. En el caso particular de la cirugía neonatal, los recién nacidos tienen un mayor riesgo de complicaciones ya que tienen menor reserva funcional y cualquier cirugía es técnicamente más exigente. Esto es especialmente crítico en recién nacidos de pretérmino. En nuestra serie, la tasa de RQNP en pacientes neonatos supera importantemente la tasa global de RQNP e incluso a la tasa de RQNP de urgencia, siendo similar a la reportada por otros autores. ${ }^{15}$

Es interesante observar que las distintas series publicadas muestran una gran dispersión de resultados y en particular las series que analizan las RQNP en adultos tienen una mayor dispersión que las series pediátricas. Al comparar los reportes, se encuentran diferencias en cuanto a la definición de RQNP, se reportan reoperaciones de diversas especialidades y subespecialidades quirúrgicas, se observan diferencias en cuanto a la complejidad de los pacientes atendidos, del método de detección y los criterios de selección, entre otros factores. Esta disparidad de criterios a la hora de definir una RQNP y el amplio rango de tasas de RQNP encontradas en ellos hace muy complejo realizar estudios comparativos entre distintos centros ${ }^{7,16}$. La norma chilena establece que la tasa de RQNP debe ser menor a un $2 \%$, sin diferenciar si la cirugía original fue electiva o de urgencia y sin distinguir la especialidad quirúrgica así como tampoco la complejidad del paciente. Una manera de mejorar el indicador RQNP incorporando estos valiosos antecedentes, sería integrando y relacionando la tasa de RNQP con el peso GRD del paciente o servicio clínico en que se trata el paciente, de tal manera de estimar el grado de complejidad del mismo y, por lo tanto, hacer comparable el indicador RQNP entre distintos servicios clínicos y hospitales.

En nuestra serie, en la gran mayoría de los casos, las causas de RQNP se debieron o a un error de técnica quirúrgica o a un error de planificación de la cirugía, lo cual coincide con lo descrito por Kroon y cols quienes demostraron que el 70\% de los casos de RQNP se deben a errores técnicos ${ }^{7}$. Además, en un número no menor de pacientes se identificó más de una causa atribuible a la RQNP. El análisis detallado de los casos clínicos, indicaciones y posibles causas de las RQNP se ha implementado paulatinamente en los servicios quirúrgicos de los hospitales de Chile desde Octubre del año 2012. Nuestro estudio muestra que los dos primeros años de implementación de la norma son aquellos con el menor número de RQNP, número que se estabiliza en los tres años sucesivos. En los primeros años de implementación, era la Unidad de Calidad del hospital quienes alertaban de los casos de RQNP; con el pasar del tiempo han sido los propios cirujanos quienes están alerta de sus casos de RQNP. Es posible, por tanto, que durante los primeros años de estudio haya habido un subregistro de pacientes reintervenidos, como consecuencia del proceso de implementación e incorporación de la normativa en los servicios clínicos. El objetivo de las reuniones de análisis de RQNP es que en el seno de un servicio clínico se identifiquen las causas de las RQNP y se propongan medidas destinadas a evitar posibles errores y disminuir las reintervenciones en el futuro. En el período estudiado, en la totalidad de los casos registrados de RQNP se realizó una reunión de análisis de la misma en nuestro hospital, cumpliendo con el $100 \%$ del indicador solicitado por el MINSAL. Esto cobra especial relevancia si consideramos que la gran mayoría de las causas de RQNP son atribuibles a errores técnicos. Creemos que las reuniones de análisis de RQNP son una valiosa herramienta de aprendizaje y mejora continua para los equipos quirúrgicos, que promueve la práctica reflexiva y otorga una retroalimentación sobre el trabajo de los cirujanos que debiera generar importantes mejoras en el quehacer médico ${ }^{3,17}$. Luego del análisis retrospectivo de cinco años de RQNP en nuestro centro, ¿qué medidas creemos necesario implementar para reducir las RQNP y favorecer una cirugía más segura y de mejor calidad para nuestros pacientes? A la luz de los resultados de nuestro trabajo y de acuerdo a Birkmeyer et $\mathrm{a}^{18}$, las medidas a implementar 
dependen del riesgo basal de la cirugía y de la frecuencia con que se realiza dicha cirugía. Para cirugías frecuentes y de bajo riesgo se recomienda implementar medidas en el proceso y medir sus resultados. En este sentido, sería recomendable protocolizar algunas cirugías. Sin embargo, la mera existencia de un protocolo o guía clínica no garantiza su adecuada implementación, por lo que también sería recomendable realizar capacitaciones periódicas a los cirujanos y medir el cumplimiento de los protocolos o guías. Por otro lado, en cirugías poco frecuentes y de alto riesgo se recomienda implementar medidas estructurales, como centralizar dichas cirugías en un sólo centro o equipo quirúrgico en particular para aumentar el volumen de cirugías de tal manera de lograr equipos de trabajo con experiencia y disminuir así la posibilidad de complicaciones ${ }^{18,19}$.

Conclusión. Las RQNP son poco frecuentes en cirugía pediátrica, excepto durante el período neonatal. Se da total cumplimiento a la normativa de reunión de análisis luego de una RQNP que indican que las causas son mayoritariamente atribuibles a la técnica o planificación quirúrgica.

\section{Responsabilidades Éticas}

Protección de personas y animales: Los autores declaran que los procedimientos seguidos se conformaron a las normas éticas del comité de experimentación humana responsable y de acuerdo con la Asociación Médica Mundial y la Declaración de Helsinki.

Confidencialidad de los datos: Los autores declaran que han seguido los protocolos de su centro de trabajo sobre la publicación de datos de pacientes.

\section{Derecho a la Privacidad y Consentimiento Informa-} do: Los autores declaran que la información ha sido obtenida de datos previos en forma anonimizada, por lo cual el Comité de Ética de Investigación en uso de sus facultades, ha eximido de la obtención de un consentimiento informado, lo cual consta en el acta respectiva.

\section{Conflicto de intereses}

Los autores declaran no tener conflicto de intereses.

\section{Referencias}

1. Santore M, Islam S. Quality improvement 101 for surgeons: Navigating the alphabet soup. Semin Pediatr Surg. 2015;24(6):26770.

2. Ministerio de Salud. Resolución Exenta $\mathrm{N}^{\circ} 1031$. Norma $\mathrm{N}^{\circ} 1$ : Establece Protocolos y Normas sobre Seguridad del paciente y Calidad de la Atención para ser aplicados por los Prestadores Institucionales Públicos y Privados. 2012. Disponible en: http://www.supersalud.gob.cl/ observatorio/671/articles-8928_recurso_1. pdf.

3. Birkmeyer J, Hamby L, Birkmeyer C, Decker M, Karon N, Dow R. Is unplanned return to the operating room a useful quality indicator in general surgery? Arch Surg. 2001;136(4):405-11.

4. Normas sobre Seguridad del Paciente y Calidad de la Atención respecto de: Reoperaciones quirúrgicas no programadas (Norma 4). Resolución Excenta $\mathrm{N}^{\circ} 1031$ del 17 de Octubre de 2012. Ministerio de Salud. Chile. Disponible en: http://www.supersalud. gob.cl/observatorio/671/articles-8928_ recurso_5.pdf.

5. Aguila A, Muñoz MA, Sepúlveda V. Experiencia en el desarrollo e implementación de la metodología de grupos relacionados por diagnóstico en un hospital universitario chileno.
Evalución a diez años de funcionamiento. Rev Med Chile 2019;147:1518-26.

6. Zapata M. Importancia del sistema GRD para alcanzar la eficiencia hospitalaria. Rev Med Clin Condes 2018; 29:347-52.

7. Kroon H, Breslau P, Lardenoye J. Can the incidence of unplanned reoperations be used as an indicator of quality of care in surgery? Am J Med Qual. 2007;22(3):198202.

8. Dimick J. What makes a "good" quality indicator? Arch Surg. 2010;145(3):295.

9. Ansari M., Collopy B. The risk of an unplanned return to the operating room in Australian hospitals. Aust N Z J Surg. 1996;66(1):10-3.

10. Khuri S, Daley J, Henderson W, et al. The National Veterans Administration Surgical Risk Study: risk adjustment for the comparative assessment of the quality of surgical care. J Am Coll Surg. 1995;180(5):519-31.

11. Bannura G, Cumsille M, Barrera A, et al. Reoperaciones precoces en cirugía colorrectal: Análisis uni y multivariado de factores de riesgo. Rev Chil Cir. 2007; 59(4):281-6.

12. Guevara O, Rubio-Romero J, Ruiz-Parra A. Unplanned reoperations: is emergency surgery a risk factor? A cohort study. J Surg Res. 2013;182(1):11-6.

13. Ramírez P, Rivas C, Scharf C, Otero H. Complicaciones post-quirúrgicas como causa de reintervención en niños sometidos a cirugía. Acta méd. Domin. 1998;20(1):1-5.

14. Kulaylat A, Rocourt D, Tsai A, et al. Understanding readmissions in children undergoing surgery: A pediatric NSQIP analysis. J Pediatr Surg. 2018;53(7):12807.

15. Boo Y, Lee E, Lee J. Comparison of surgical outcomes among infants in neonatal intensive care units treated by pediatric surgeons versus general surgeons: The need for pediatric surgery specialists. J Pediatr Surg. 2017;52(11):1715-7.

16. McLaughlin N, Jin P, Martin N. Assessing early unplanned reoperations in neurosurgery: opportunities for quality improvement. J Neurosurg. 2015;123(1):198-205.

17. Ivers $\mathrm{N}$, Jamtvedt $\mathrm{G}$, Flottorp $\mathrm{S}$, et al. Audit and feedback: effects on professional practice and healthcare outcomes. Cochrane Database Syst Rev. 2012;13(6):CD000259.

18. Birkmeyer J, Dimick J, Birkmeyer N. Measuring the quality of surgical care: structure, process, or outcomes? J Am Coll Surg. 2004;198(4):626-32.

19. Lee Hall B, Hsiao E, Majercik S, Hirbe M, Hamilton B. The Impact of Surgeon Specialization on Patient Mortality. Examination of a Continuous Herfindahl-Hirschman Index. Ann Surg 2009;249:708-16. 